\title{
Radiation Induced Cerebral Microbleeds in Pediatric Patients with Brain Tumors Treated with Proton Radiotherapy
}

Stephen F. Kralik, MD ${ }^{1}$

Todd R. Mereniuk, MD/PhD ${ }^{2}$

Laurent Grignon, $\mathrm{MD}^{1}$

Chie-Schin Shih, MD ${ }^{3}$

Chang Y. Ho, MD ${ }^{1}$

Whitney Finke, $\mathrm{MD}^{1}$

Peter W. Coleman, $\mathrm{MD}^{2}$

Gordon A. Watson, $\mathrm{MD} / \mathrm{PhD}^{2}$

Jeffrey Buchsbaum, MD ${ }^{4}$

${ }^{1}$ Department of Radiology and Imaging Sciences, Indiana University School of Medicine, Indianapolis, Indiana

${ }^{2}$ Department of Radiation Oncology, Simon Cancer Center, Indiana University

${ }^{3}$ Department of Pediatrics, Hematology/Oncology Section, Indiana University School of Medicine, Indianapolis, Indiana

${ }^{4}$ Department of Radiation Oncology, Indiana University School of Medicine, Indianapolis, Indiana

Conflict of interest: None.

Funding: No source of funding was used for this research.

Correspondence to:

Stephen F. Kralik M.D.

Department of Radiology and Imaging Sciences, Indiana University School of Medicine,

714 N. Senate Avenue

This is the author's manuscript of the article published in final edited form as:

Kralik, S. F., Mereniuk, T. R., Grignon, L., Shih, C.-S., Ho, C. Y., Finke, W., .. Buchsbaum, J. (2018). Radiation Induced Cerebral Microbleeds in Pediatric Patients with Brain Tumors Treated with Proton Radiotherapy. International Journal of Radiation Oncology*Biology*Physics. https://doi.org/10.1016/j.ijrobp.2018.07.2016 
Indianapolis, Indiana, 46202

Tel:1 (317) 963-7121

Fax: 1 (317) 948-8383

E-mail: steve.kralik@gmail.com 


\title{
Radiation Induced Cerebral Microbleeds in Pediatric Patients with Brain Tumors Treated with Proton Radiotherapy
}

\author{
Abstract \\ Purpose \\ Proton beam radiotherapy (PBT) has been increasingly utilized to treat pediatric brain tumors, \\ however, limited information exists regarding radiation induced cerebral microbleeds (CMBs) \\ among these patients. The purpose was to evaluate the incidence, risk factors, and imaging \\ appearance of CMBs in pediatric patients with brain tumors treated with PBT.
}

\section{Methods}

A retrospective study was performed on 100 pediatric patients with primary brain tumors treated with PBT. CMBs were diagnosed by examining serial MRIs including susceptibility-weighted imaging. Radiation therapy plans were analyzed to determine doses to individual CMBs. Clinical records were used to determine risk factors associated with the development of CMBs in these patients.

\section{Results}

The mean age at time of PBT was 8.1 years. The median follow-up duration was 57 months. The median time to development of CMBs was 8 months (mean 11 months; range 3-28 months). The percentage of patients with CMBs was $43 \%, 66 \%, 80 \%, 81 \%, 83 \%$, and $81 \%$ at 1-year, 2 -years, 3 -years, 4-year, 5-years, and greater than 5 years from completion of proton radiotherapy. The majority (87\%) of CMBs were found in areas of brain exposed to $\geq 30 \mathrm{~Gy}$. Risk factors included maximum radiotherapy dose $(\mathrm{P}=0.001)$, percentage and volume of brain exposed to $\geq 30 \mathrm{~Gy}$ $(\mathrm{P}=0.0004 ; \mathrm{P}=0.0005)$, and patient age at time of $\mathrm{PBT}(\mathrm{P}=0.0004)$. Chemotherapy was not a significant risk factor $(\mathrm{P}=0.35)$. No $\mathrm{CMBs}$ required surgical intervention.

\section{Conclusion}

CMBs develop in a high percentage of pediatric patients with brain tumors treated with proton radiotherapy within the first few years following treatment. Significant risk factors for development of CMBs include younger age at time of PBT, higher maximum radiotherapy dose, and higher percentage and volume of brain exposed to $\geq 30 \mathrm{~Gy}$. These findings demonstrate similarities with CMBs that develop in pediatric brain tumor patients treated with photon radiotherapy.

Abbreviations: CMBs: cerebral microbleeds; DWI: diffusion-weighted; FLAIR: fluid attenuated inversion recovery; GRE: gradient echo; MPRAGE: magnetization prepared rapid acquisition gradient echo; PBT: proton beam therapy; SWI: susceptibility-weighted imaging; TSE: turbo spin echo 


\section{Introduction}

Radiotherapy is one of the primary therapies used to treat pediatric brain tumors. Adverse effects of radiotherapy on the brain include radiation necrosis, atrophy, gliosis, telangiectasia, microhemorrhages, cavernous malformations, and large vessel vasculopathy. MRIs performed following cranial radiotherapy frequently detect small parenchymal lesions which demonstrate susceptibility artifact and have been termed radiation-induced cerebral microbleeds (CMBs). CMBs have been found to be associated with worse executive function in pediatric brain tumor survivors treated with radiotherapy and among patients with nasopharyngeal carcinoma treated with radiotherapy. ${ }^{1,2}$ Among patients without history of cranial radiotherapy, CMBs have also been associated with cognitive impairment in Alzheimers disease, stroke, vascular dementia, small vessel ischemic disease and increasing age. ${ }^{3-10}$

Compared with conventional (photon) radiation therapy, proton beam therapy (PBT) offers the advantages of the absence of an exit dose, a highly conformal dose distribution, and a reduced radiation dose to adjacent normal tissue. ${ }^{11}$ Therefore, potential benefits of PBT in patients with pediatric brain tumors may include the reduction of negative long-term effects of radiation, such as cognitive deficits, endocrine abnormalities, vascular abnormalities, and secondary malignancies. ${ }^{12}$ PBT has been used increasingly to treat pediatric brain tumors, including craniopharyngiomas, ependymomas, germinomas, and medulloblastomas; however, limited information exists regarding CMBs following PBT. ${ }^{13-16}$ Although CMBs can be diagnosed on histopathology, the preferred method for detection of CMBs is with a brain MRI. Gradient echo (GRE) imaging and/or susceptibility-weighted imaging (SWI) MRI sequences are necessary for detection of CMBs because these MRI sequences have been developed to increase the conspicuity of the blood products that cause CMBs. SWI represents the most current and 
advanced MRI sequence that is commercially available for detection of CMBs and has been shown to be much more sensitive than GRE for detection of CMBs. ${ }^{17-19}$ Therefore, clinical research investigating the formation of CMBs should include SWI to allow the best possible imaging assessment.

The purpose of this research was to evaluate the incidence, imaging appearance, and risk factors for radiation induced CMBs among pediatric patients with brain tumors treated with PBT using MRI with SWI.

\section{Materials and Methods}

Following institutional review board approval, a retrospective study was performed from January 2010 to January 2017 on pediatric patients age $\leq 18$ with primary brain tumors who were treated with PBT. All patients were either those with a newly diagnosed primary brain tumor who were subsequently treated with PBT or those who had low-grade gliomas without prior treatment with radiation therapy who demonstrated tumor progression on chemotherapy necessitating treatment with PBT. Therefore, patients were excluded if there was any history of treatment with photon radiation therapy, including before PBT, concurrent with PBT, or after PBT. Patients were also excluded if there was more than one course of PBT. Patients without a minimum 3-month follow-up brain MRI following completion of PBT were excluded, including if death occurred before 3 months. Patients were also excluded if there was a clinical history of hypertension, known coagulopathy, collagen vascular disease or other medical history that would predispose to intracranial hemorrhage. PBT treatment doses followed the standard of care in the United States at a Children's Oncology Group treatment center.

Brain MRI consisted of imaging performed with 1.5T or 3T (Avanto and Verio; Siemens, 
Erlangen, Germany) MR imaging units with standard MRI sequences at our institution including: sagittal T1-weighted (T1W) magnetization prepared rapid acquisition gradient echo (MPRAGE), axial T2-weighted (T2W) turbo spin echo (TSE), axial fluid attenuated inversion recovery (FLAIR), axial diffusion-weighted (DWI), axial susceptibility weighted imaging (SWI), coronal T1W TSE post-contrast with fat saturation, and axial 3D T1W MPRAGE post contrast pulse sequences. Post-contrast imaging was performed in all patients after $0.1-\mathrm{mmol} / \mathrm{kg}$ intravenous administration of gadobenate dimeglumine (MultiHance; Bracco Diagnostics, Princeton, New Jersey). SWI was performed with the following parameters: $1.5 \mathrm{~T}$ flip angle 15 , TR 49, TE 40, slice thickness; 3.0T Flip angle 30, TR 27, TE 20, slice thickness. In the majority of patients, brain MRIs were performed approximately every 1-3 months for 2-3 years, then every 6-12 months with shorter or longer follow-up depending on clinical need to assess tumor stability, clinical symptoms, and/or other complications including radiation necrosis. Patients were included if one or more of the follow-up brain MRIs included SWI. Patients were excluded if none of the follow-up brain MRIs included an SWI sequence. Because the SWI sequence was not available on all MRI scanners at our institution dating back to 2010, some patients did not have SWI on every follow up brain MRI. The number of patients who had SWI available for review at each yearly interval was indicated in the results by the total number of patients at each time point. Only patients with SWI on every follow-up brain MRI were used to determine the time to development of CMBs.

A fellowship-trained, board-certified neuroradiologist (S.K.) with certificate of added qualification in neuroradiology independently evaluated all brain MRIs of included patients. SWI sequences in conjunction with the other MRI sequences were evaluated for: presence, number, and largest size of CMBs or cavernous malformation. Clinical data included patient age at time 
of PBT, patient gender, tumor pathology from surgical resection or biopsy, tumor location, total cranial radiation dose, chemotherapy, timing from completion of PBT to imaging diagnosis of CMBs, and surgical intervention for CMBs. CMBs were defined similarly to consensus MRI criteria described by Greenberg et al as an intraparenchymal small (5 $\mathrm{mm}$ or less) round or ovoid hypointensity on SWI imaging that did not correspond to vessels, tumor, border the surgical resection site, or expected location of mineralization and was not hyperintense on $\mathrm{T} 1 \mathrm{~W}$ or $\mathrm{T} 2 \mathrm{~W}$ imaging. ${ }^{20}$ To distinguish CMBs from a cavernous malformation, a cavernous malformation was defined as a round or ovoid hypointensity on SWI that demonstrated hyperintense T1 W and/or T2W appearance. ${ }^{20}$

To determine the specific amount of radiation received by CMBs, radiation dosimetric treatment plans were either overlaid and aligned with SWI MRI sequence using Eclipse image registration software (v13.7, Varian medical systems) to match voxel intensity values or locations of CMBs on SWI images were cross referenced to location on the treatment plan by side by side comparison. Overlaid MRI images were first registered to the planning CT scan using automatic match based on bony anatomy, after which the alignment was manually adjusted to correct for any errors. Dose information for individual CMBs were then recorded by adjusting the dose slider in $10 \mathrm{~Gy}$ increments. Based on a prior study indicating majority of CMBs occur at $>30 \mathrm{~Gy}$, the percentage and overall volume of brain receiving $\geq 30 \mathrm{~Gy}$ of total dose was calculated for each patient using the treatment planning software which determined total volumes of the contoured brain, and the dose volume histogram corresponding to $30 \mathrm{~Gy}$ dose level. ${ }^{1}$ This information was then used for statistical analysis.

Statistical analysis of risk factors for CMBs including age at time of PBT, chemotherapy, percentage and volume of brain exposed to $\geq 30 \mathrm{~Gy}$, and maximum proton radiotherapy dose 
was performed by using an unpaired t-test, Fisher exact test, or Pearson correlation coefficient where appropriate and a $\mathrm{P}$ value of $<0.05$ was considered statistically significant. Statistics were performed using Graphpad Prism 7 Statistical Software (La Jolla, CA).

\section{Results}

A total of 100 pediatric patients met inclusion criteria while 26 patients were excluded. Mean age at time of PBT was 8.1 years (range 0.75-18 years), and male:female ratio was 63:37. Median follow up duration was 57 months (mean 52 months, range 7-116 months). Patient characteristics are seen in Table 1.

The median time to development of CMBs was 8 months (mean 11 months; range 3-28 months). The percentage of patients with CMBs was $43 \%$ (16/37), 66\% (27/41), 80\% (20/25), $81 \%$ (26/32), 83\% (35/42), and 81\% (29/36) at 1-year, 2-years, 3-years, 4-year, 5-years, and greater than 5 years from completion of proton radiotherapy (Figure 1). The median number and range of CMBs per patient was $6(0-49)$ at 1-year, $6(0-63)$ at 2-years, $6(0-72)$ at 3 -years, $7(0-$ $130)$ at 4-years, $8(0-136)$ at 5 -years, and $11(0-136)$ at greater than 5 years from completion of proton radiotherapy. (Figure 2). The median size of CMBs was $0.2 \mathrm{~cm}$ (range 0.1-0.5 cm). CMBs were detected in all areas of the brain, specifically the brainstem, cerebellum, thalami, basal ganglia, and cerebral hemispheres. No CMBs demonstrated resolution on follow up imaging. No $\mathrm{CMBs}$ required surgical intervention. Four patients (4\%) demonstrated imaging appearance consistent with a cavernous malformation which developed at a median time of 46 months (range 14-72 months). Representative examples of CMBs and cavernomas are seen in Figures 3 and 4. 
Evaluation of radiation dose to individual CMBs demonstrated that $87 \%$ of CMBs occurred in areas of brain exposed to $\geq 30 \mathrm{~Gy}$ (32.8\% for $30-40 \mathrm{~Gy}, 18.8 \%$ for $40-50 \mathrm{~Gy}, 35.7 \%$ for $>50 \mathrm{~Gy})$ and $13 \%$ exposed to $<30 \mathrm{~Gy}(4.1 \%$ for $<10 \mathrm{~Gy}, 0.6 \%$ for $10-20 \mathrm{~Gy}$, and $7.9 \%$ for 20-30 Gy) (Figure 5). There were statistically significant differences among patients without CMBs and patients with CMBs in the percentage of brain exposed to $\geq 30 \mathrm{~Gy}(10.1 \%$ vs $42.1 \%$; $\mathrm{P}=0.0004)$ and volume of brain exposed to $\geq 30 \mathrm{~Gy}(133 \mathrm{cc}$ vs $601 \mathrm{cc} ; \mathrm{P}=0.0005)$. There was a statistically significant positive correlation between the number of CMBs and percentage of brain exposed to $\geq 30 \mathrm{~Gy}(\mathrm{r}=0.39 ; \mathrm{P}=0.0002)$.

There was a statistically significant difference in the mean age at time of PBT between patients with and without $\mathrm{CMBs}(6.9$ vs 10.6 years; $\mathrm{P}=0.0004)$. There was a statistically significant difference in maximum radiotherapy dose between patients with and without CMBs (55.9 versus $51.6 \mathrm{~Gy} ; \mathrm{P}=0.001$ ). Chemotherapy was not a statistically significant risk factor $(\mathrm{P}=0.35)$ for development of CMBs between patients treated with chemotherapy versus without chemotherapy.

\section{Discussion}

In this study, approximately $80 \%$ of pediatric brain tumor patients treated with PBT developed CMBs indicating a high frequency in this patient population. In comparison, the incidence of CMBs in pediatric patients with primary brain tumors treated with conventional/photon radiotherapy has been reported to occur over a wide range of frequencies of approximately $3-80 \% .^{1,21-23}$ The high incidence found in our study is similar to a study by Passos et al of 100 childhood primary central nervous system tumors treated with photon radiotherapy, where CMBs were identified in $80.6 \%$ of patients, however, Passos et al also reported cavernous 
malformations in $52.8 \%$ of patients which is different from the $4 \%$ in our study. ${ }^{23}$ A potential explanation for differences in cavernous malformations may be a difference in follow up duration of approximately 4.5 years in this study compared to 11 years in the study from Passos et al. ${ }^{23}$ The wide range in incidence of CMBs and cavernous malformations following photon radiotherapy is also very dependent on whether GRE, SWI, or neither MRI sequence was used to detect them. Careful review of the research study methods is necessary as studies reporting CMBs using GRE for detection will tend to demonstrate a lower incidence of CMBs compared to studies using SWI. For example, Burn et al reported an incidence of 3.4\% but did not use GRE or SWI for detection of CMBs, while Roddy et al reported an incidence of $48.8 \%$ but used a combination of GRE and SWI imaging. ${ }^{1,21}$ Undoubtedly, the use of SWI to detect CMBs in this study results in an incidence that is at the high end for the range reported with photon radiotherapy. We believe using SWI, rather than GRE, to detect CMBs in our study is a major advantage because it represents the most sensitive of the currently available MRI technology clinically available and allows the best imaging depiction of what is present histopathologically. If MRI technology advances from SWI to an even greater level of sensitivity for detection of CMBs, future studies would be necessary to determine if the incidence is even greater in these patients.

CMBs appeared at a median time of 8 months following completion of PBT. Similar to our results, Peters et al demonstrated that in children treated with photon radiotherapy, CMBs may appear as early as 5 months and much more variable rate of formation and faster rates than observed in adults. ${ }^{24}$ Tanino et al demonstrated a wider time range to development of CMBs of 3 months to 9 years (mean, 33 months), however this study included children and adults with a mean age of patients of 49 years (range 13-78 years). ${ }^{25}$ These results suggest that development of 
CMBs is affected by patient age at time of radiotherapy.

In this study, we have identified that volume of brain exposed to $\geq 30 \mathrm{~Gy}$, higher maximum PBT dose, and younger age at time of PBT are risk factors for development of CMBs. These risk factors likely in part explain why some patients do not develop CMBs. Our results demonstrated that $87 \%$ of CMBs occurred in areas exposed to $\geq 30$ Gy which is similar to $91 \%$ occurring in areas receiving > 30 Gy reported by Roddy et al who evaluated patients treated with photon radiotherapy. Our results are not completely similar however to Tanino et al who reported in a smaller study of 34 pediatric and adult patients treated with photon radiotherapy that all CMBs were in areas exposed to $>25 \mathrm{~Gy}$. The percentage of patients with CMBs stabilized at 3 years following completion of PBT indicating if a patient is to develop CMBs, it will most likely occur within 3 years from completion of proton radiotherapy. However, the number of CMBs per patient continued to increase over time. Similar to these findings, Lupo et al demonstrated that the rate of CMBs increased after 2 years following radiotherapy for adult patients with high grade gliomas. ${ }^{26} \mathrm{We}$ also demonstrate that younger age at time of PBT was a risk factor for development of CMBs. Similarly, Passos et al demonstrated that age at time of radiotherapy was a risk factor for development of CMBs. ${ }^{23}$ Lastly, in this study, chemotherapy was not associated with development of CMBs which is similar to the previous study by Passos et al. ${ }^{23}$

This study has a few important limitations. First, pathologic correlation to confirm these lesions represent CMBs was not performed, and it is the presumption that these CMBs are secondary to radiotherapy. It is not standard clinical care to biopsy these areas, and prior research has demonstrated that $\mathrm{CMBs}$ are not seen in patients treated only with chemotherapy. ${ }^{23,27-28}$ One potential explanation for the association between radiotherapy and CMBs is that vascular 
endothelial growth factor is increased following radiotherapy. ${ }^{29-31}$ There are rare genetic causes of multiple cavernomas in which patients have numerous cavernomas although this is unlikely in our patient population. Genetic testing was not performed in these patients given that none of these patients had multiple cavernomas prior to PBT which would be expected with a familial cavernoma syndrome, as well as the fact that a high percentage of patients $(\sim 80 \%)$ develop CMBs after PBT indicating an unlikelihood for an etiology of a rare genetic syndrome. However, it is possible that there are unknown genetic differences among these patients that predispose some patients to being more susceptible to CMB formation following exposure to radiotherapy. While CMBs can also be caused by systemic hypertension, amyloid angiopathy, or diffuse axonal injury, our patient population does not support these as potential etiologies which is why we believe the CMBs in these patients are related to PBT. Second, our criteria for including patients with SWI imaging resulted in some patients that could not be evaluated at every time interval from completion of PBT. SWI has been replacing GRE at many institutions and represents the most sensitive MRI technique for the detection of hemorrhage currently available for clinical use. Several factors can affect the image quality and sensitivity of the SWI sequence including echo time, flip angle, slice thickness, and magnetic field strength, however, the optimal parameters have yet to be determined. ${ }^{4}$ Because this was a retrospective study, we could not control for patients with SWI sequence performed at either 1.5T and 3T. We chose to include all patients with SWI performed at $1.5 \mathrm{~T}$ or $3 \mathrm{~T}$ and acknowledge this could impact our results. Lastly, we did not assess cognitive function in these patients. Because of the retrospective nature of this study, not all patients had neuropsychology testing, and the neuropsychology testing was neither performed at a consistent time following PBT nor standardized to provide an adequate analysis. We anticipate a similar negative impact on cognitive function associated with CMBs 
which has been previously reported. ${ }^{1,2}$ Future research would be useful in correlating CMBs with neuropsychology testing, and longer follow-up duration to assess development of cavernous malformations.

\section{Conclusion}

CMBs are seen with high frequency and develop in the first few years following PBT for brain tumors in pediatric patients. Risk factors for development of CMBs include younger age at time of PBT, percentage and volume of brain exposed to $\geq 30 \mathrm{~Gy}$, and higher maximum radiotherapy dose. These findings demonstrate similarities with CMBs that develop in pediatric brain tumor patients treated with photon radiotherapy.

\section{References:}

1. Roddy E, Sear K, Felton E, et al. Presence of cerebral microbleeds is associated with worse executive function in pediatric brain tumor survivors. Neuro Oncol. 2016 Nov; 18(11):1548-1558

2. Shen Q, Lin F, Rong X, et al. Temporal Cerebral Microbleeds Are Associated With Radiation Necrosis and Cognitive Dysfunction in Patients Treated for Nasopharyngeal Carcinoma. Int J Radiat Oncol Biol Phys. 2016 Apr 1;94(5):1113-20

3. Fazekas F, Kleinert R, Roob G, et al. Histopathologic analysis of foci of signal loss on gradient-echo T2*-weighted MR images in patients with spontaneous intracerebral hemorrhage: evidence of microangiopathy-related microbleeds. AJNR Am J Neuroradiol. 1999 Apr;20(4):637-642

4. Charidimou A, Krishnan A, Werring DJ, et al. Cerebral microbleeds: a guide to detection and clinical relevance in different disease settings. Neuroradiology 2013 Jun;55(6): 655674

5. Werring DJ, Gregoire SM, Cipolotti L. Cerebral microbleeds and vascular cognitive impairment. J Neurol Sci. 2010 Dec 15;299(1-2):131-135

6. Werring DJ, Frazer DW, Coward LJ, et al. Cognitive dysfunction in patients with cerebral microbleeds on T2*-weighted gradient-echo MRI. Brain 2004 Oct;127(Pt 10):2265-2275

7. Pettersen JA, Sathiyamoorthy G, Gao FQ, et al. Microbleed topography, leukoaraiosis, and cognition in probable Alzheimer disease from the Sunnybrook dementia study. Arch Neurol 2008 Jun;65(6):790-795 
8. Gregoire SM, Scheffler G, Jager HR, et al. Strictly lobar microbleeds are associated with executive impairment in patients with ischemic stroke or transient ischemic attack. Stroke 2013 May;44(5):1267-1272

9. Van der Flier WM, Cordonnier C. Microbleeds in vascular dementia: Clinical aspects. Exp Gerontol 2012 Nov;47(11):853-857

10. Patel B, Lawrence AJ, Chung AW, et al. Cerebral microbleeds and cognition in patients with symptomatic small vessel disease. Stroke 2013 Feb;44(2):356-361

11. Levin WP, Kooy H, Loeffler JS, et al. Proton beam therapy. Br J Cancer 2005 Oct 17;93(8):849-854

12. Boehling NS, Grosshans DR, Bluett JB, et al. Dosimetric comparison of threedimensional conformal proton radiotherapy, intensity-modulated proton therapy, and intensity-modulated radiotherapy for treatment of pediatric craniopharyngiomas. Int $\mathbf{J}$ Radiat Oncol Biol Phys 2012 Feb 1;82(2):643-652

13. Howell RM, Giebeler A, Koontz-Raisig W, et al. Comparison of therapeutic dosimetric data from passively scattered proton and photon craniospinal irradiations for medulloblastoma. Radiat Oncol 2012 Jul 24;7:116

14. MacDonald SM, Safai S, Trofimov A, et al. Proton radiotherapy for childhood ependymoma: initial clinical outcomes and dose comparisons. Int J Radiat Oncol Biol Phys 2008 Jul 15;71(4):979-986

15. Jimenez RB, Sethi R, Depauw N, et al. Proton radiation therapy for pediatric medulloblastoma and supratentorial primitive neuroectodermal tumors: outcomes for very young children treated with upfront chemotherapy. Int J Radiat Oncol Biol Phys 2013 Sep;87(1):120-126

16. MacDonald SM, Trofimov A, Safai S, et al. Proton radiotherapy for pediatric central nervous system germ cell tumors: early clinical outcomes. Int J Radiat Oncol Biol Phys 2011 Jan;79(1):121-29

17. Vernooij MW, Ikram MA, Wielopolski PA, et al. Cerebral microbleeds: accelerated 3D T2*-weighted GRE MR imaging versus conventional 2D T2*-weighted GRE MR imaging for detection. Radiology 2008 Jul;248(1):272-277

18. Cheng AL, Batool S, McCreary CR, et al. Susceptibility-weighted imaging is more reliable than T2*-weighted gradient-recalled echo MRI for detecting microbleeds. Stroke 2013 Oct;44(10):2782-86

19. Nandigam RN, Viswanathan A, Delgado P, et al. MR Imaging Detection of Cerebral Microbleeds: Effect of Susceptibility-Weighted Imaging, Section Thickness, and Field Strength. AJNR Am J Neuroradiol. 2009 Feb;30(2):338-43

20. Greenberg SM, Vernooij MW, Cordonnier C, et al. Cerebral microbleeds: a field guide to their detection and interpretation. Lancet Neurol. 2009 Feb:8(2):165-174

21. Burn S, Gunny R, Phipps K, et al. Incidence of cavernoma development in children after radiotherapy for brain tumors. J Neurosurg. 2007 May;106(5 Suppl):379-83

22. Lew SM, Morgan JN, Psaty E, et al. Cumulative incidence of radiation-induced cavernomas in long-term survivors of medulloblastoma. J Neurosurg. 2006 Feb;104(2 Suppl):103-7

23. Passos J, Nzwalo H, Marques J, et al. Late cerebrovascular complications after radiotherapy for childhood primary central nervous system tumors. Pediatric Neurology 2105 Sep;53(3):211-215

24. Peters S, Pahl R, Claviez A, et al. Detection of irreversible changes in susceptibility- 
weighted images after whole-brain irradiation of children. Neuroradiology. 2013 Jul;55(7):853-859

25. Tanino T, Kanasaki Y, Tahara T, et al. Radiation-Induced Microbleeds after Cranial Irradiation: Evaluation by Phase-Sensitive Magnetic Resonance Imaging with 3.0 Tesla. Yonago Acta Med. 2013 Mar;56(1):7-12

26. Lupo JM, Molinaro AM, Essock-Burns E, et al. The effects of anti-angiogenic therapy on the formation of radiation-induced microbleeds in normal brain tissue of patients with glioma. Neuro Oncol. 2016 Jan;18(1):87-95

27. Lupo JM, Chuang CF, Chang SM, et al. 7-Tesla susceptibility-weighted imaging to assess the effects of radiotherapy on normal-appearing brain in patients with glioma. Int $\mathbf{J}$ Radiat Oncol Biol Phys. 2012 Mar 1;82(3):e493-e500

28. Koike S, Aida N, Hata M, et al. Asymptomatic radiation-induced telangiectasia in children after cranial irradiation: frequency, latency, and dose relation. Radiology. 2004 Jan;230(1):93-99

29. Faraci M, Morana G, Bagnasco F, et al. Magnetic resonance imaging in childhood leukemia survivors treated with cranial radiotherapy: a cross sectional, single center study. Pediatr Blood Cancer. 2011 Aug;57(2):240-6

30. Jain R, Robertson PL, Gandhi D, et al. Radiation-induced cavernomas of the brain. AJNR Am J Neuroradiol. 2005 May;26(5):1158-62

31. Nimjee SM, Powers CJ, Bulsara KR. Review of the literature on de novo formation of cavernous malformations of the central nervous system after radiation therapy. Neurosurg Focus. 2006 Jul 15;21(1):e4 
Figure 1. Percentage of patients with CMBs versus time from completion of PBT

Figure 2. Median number of CMBs versus time from completion of PBT

Figure 3. Example of an SWI MRI image overlaid onto the radiation dosimetric treatment plan of a 7 year old with multifocal PNET treated with craniospinal PBT. CMBs (arrows) are demonstrated within varying radiation doses in cGy as indicated by the color gradient.

Figure 4. Formation of CMBs and a cavernous malformation following PBT. A 3 year-old with history of medulloblastoma treated with PBT. Axial SWI (A) demonstrates multiple cerebral microbleeds in both cerebral hemispheres in addition to a right frontal lobe cavernous malformation which demonstrates hyperintense signal on T1W (B) and T2W (C) images. 
Table 1: Patient Characteristics

\begin{tabular}{l|l}
\multicolumn{1}{c}{ Category } & \multicolumn{1}{c}{ Characteristics } \\
\hline Age & Mean 8.1 years (range 1.5-18 years) \\
Tumor Pathology & Male/Female 63:37 \\
Medulloblastoma/PNET 28 & Ependymoma 19 \\
Craniopharyngioma 17 & Pilocytic/Pilomyxoid astrocytoma 9 \\
Germinoma 7 \\
GBM/Anaplastic Astrocytoma 4 \\
Total cranial radiation dose & ATRT 3 \\
Brainstem glioma 5 \\
Mature teratoma 3 \\
Pineal Parencymal Tumor 2 \\
Pineoblastoma 1 \\
Pituitary adenoma 1 \\
Meningioma 1 \\
Mean 54.6 Gy (range 30-59.4 Gy) \\
Supratentorial 50 \\
- Sella/Suprasellar/Optic Pathway 27 \\
- Cerebral Hemisphere 12 \\
- Pineal 11 \\
Infratentorial 48 \\
-Cerebellar/4
\end{tabular}

Abbreviations: PNET: primitive neuroectodermal tumor; GBM: glioblastoma multiforme; ATRT: atypical teratoid rhabdoid tumor 


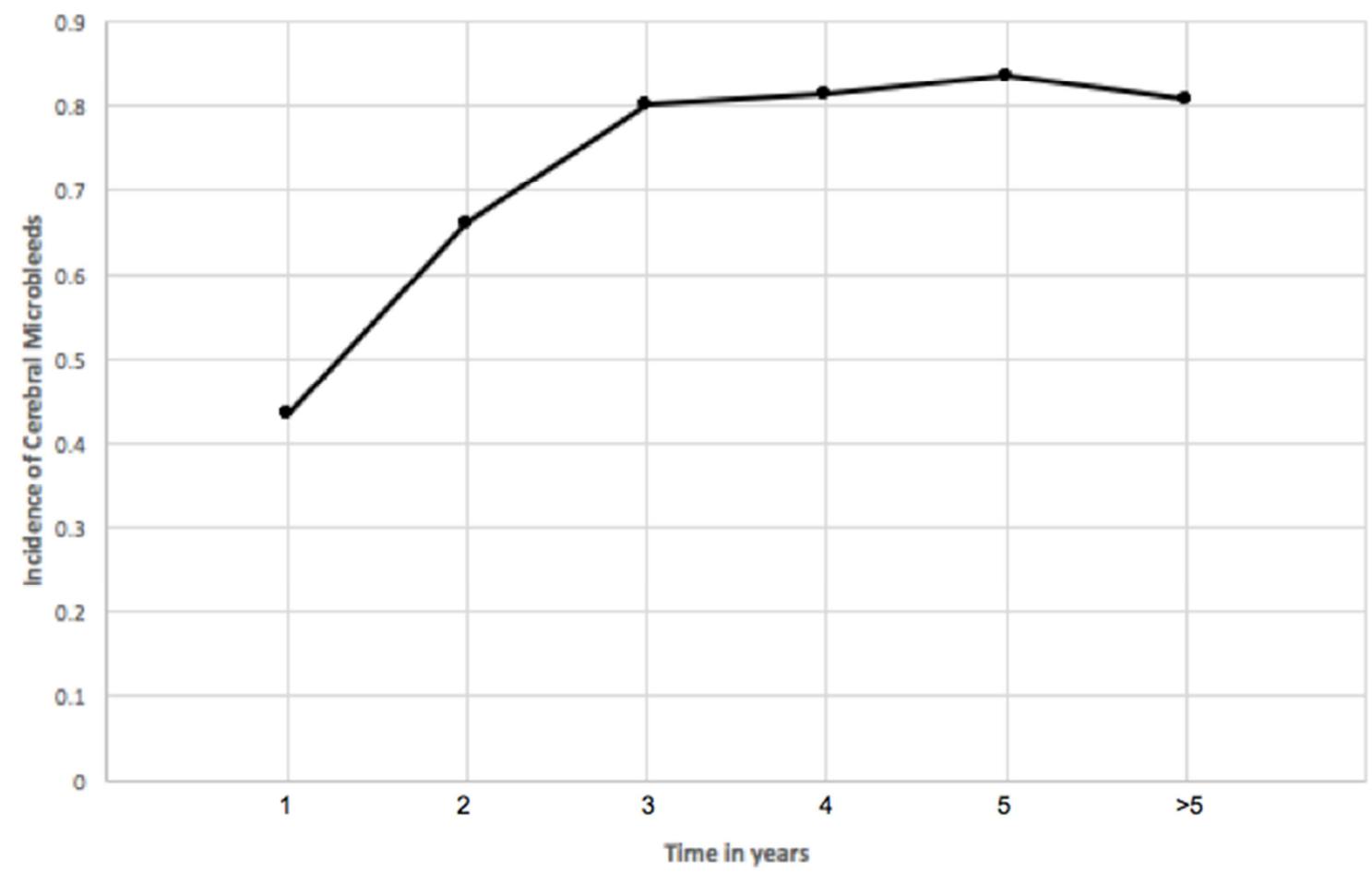

Number at risk: 97 


\section{ACCEPTED MANUSCRIPT}

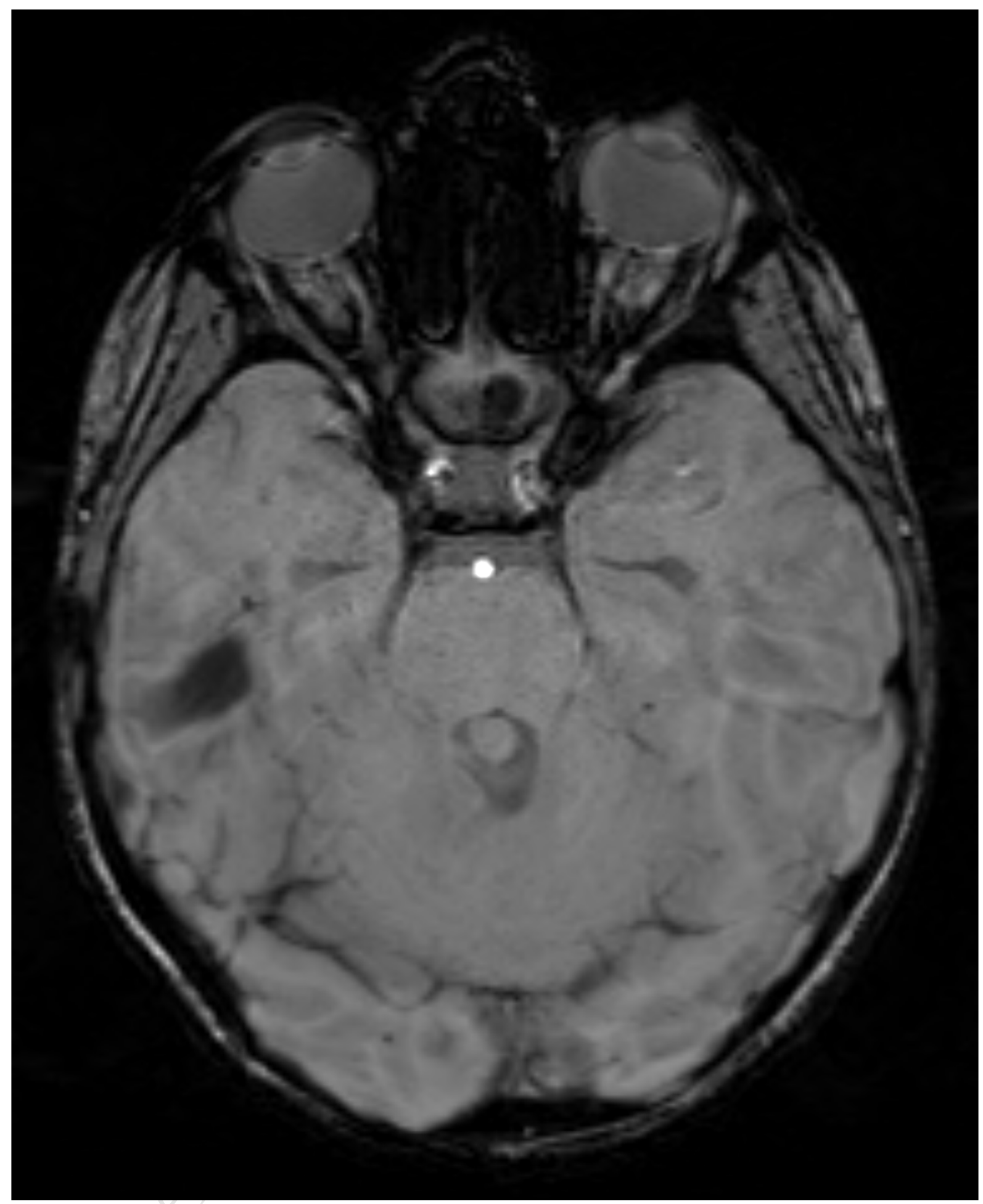




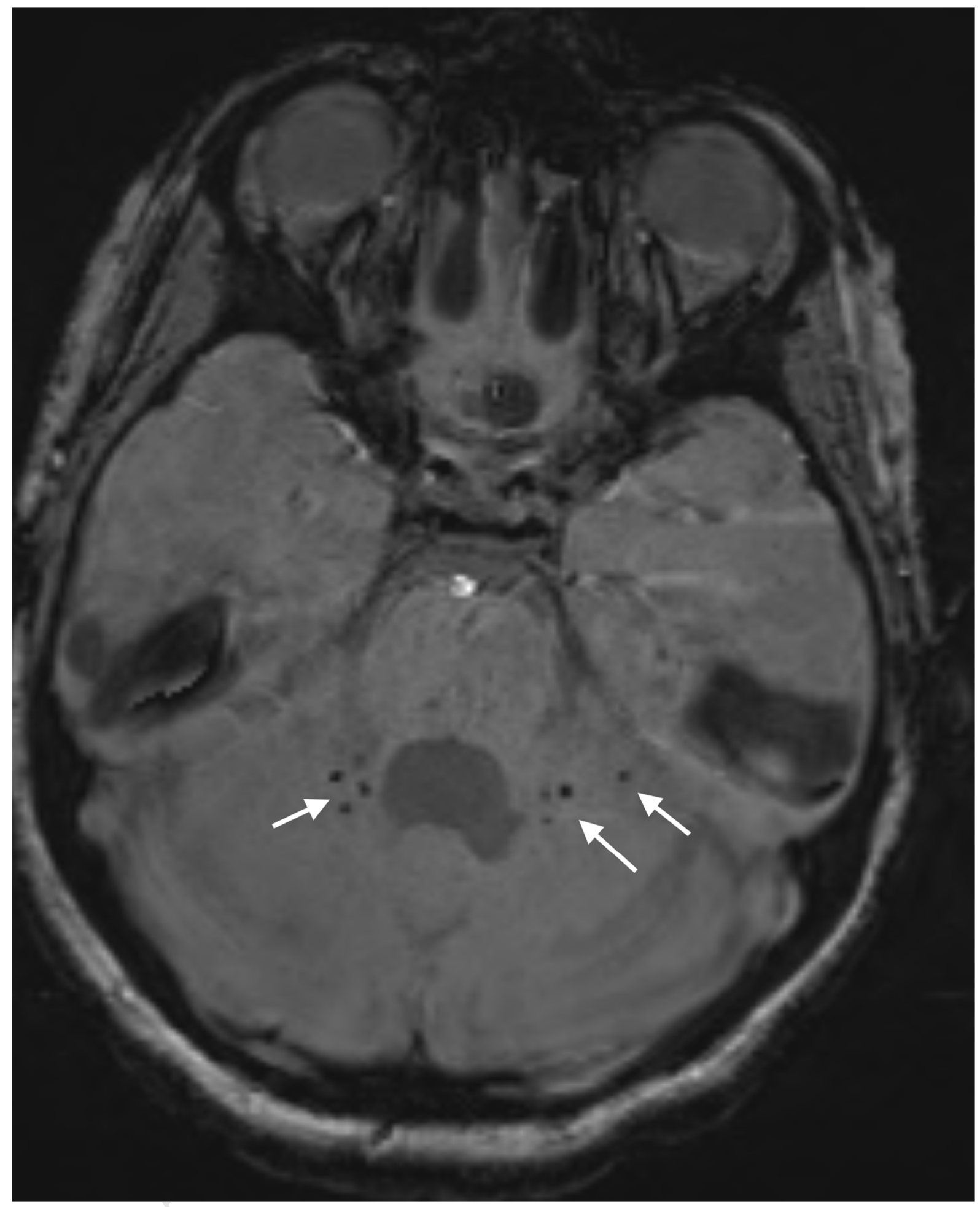




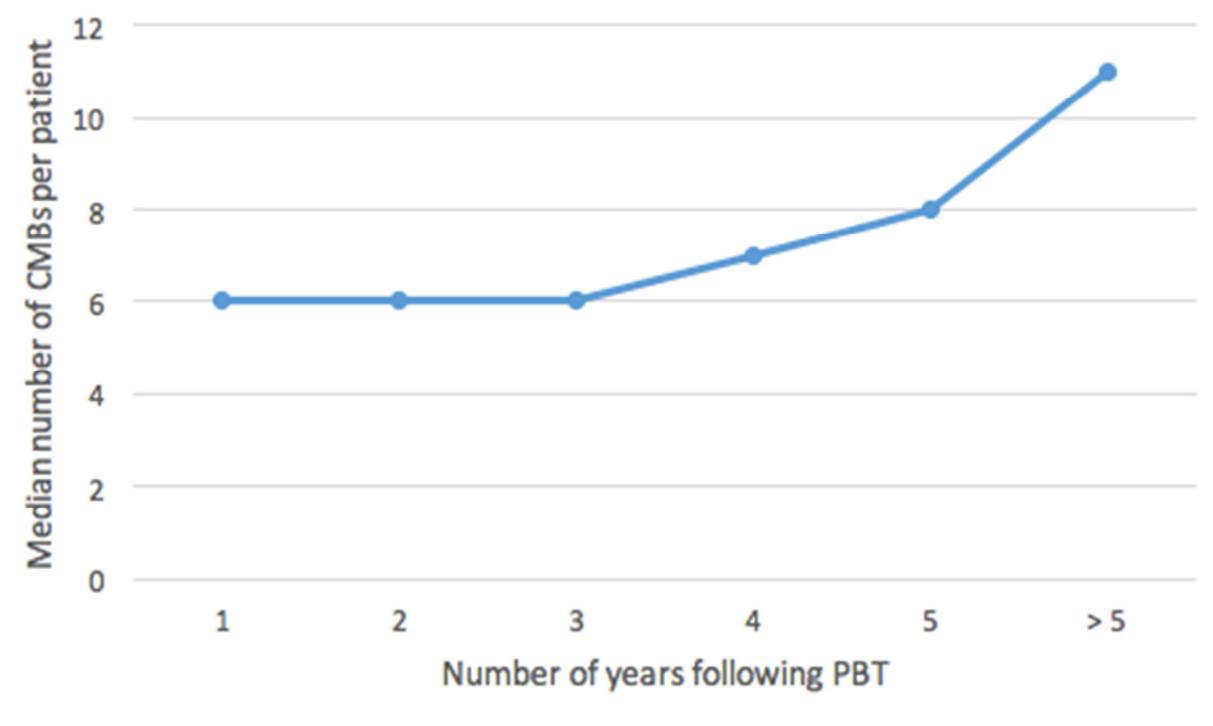




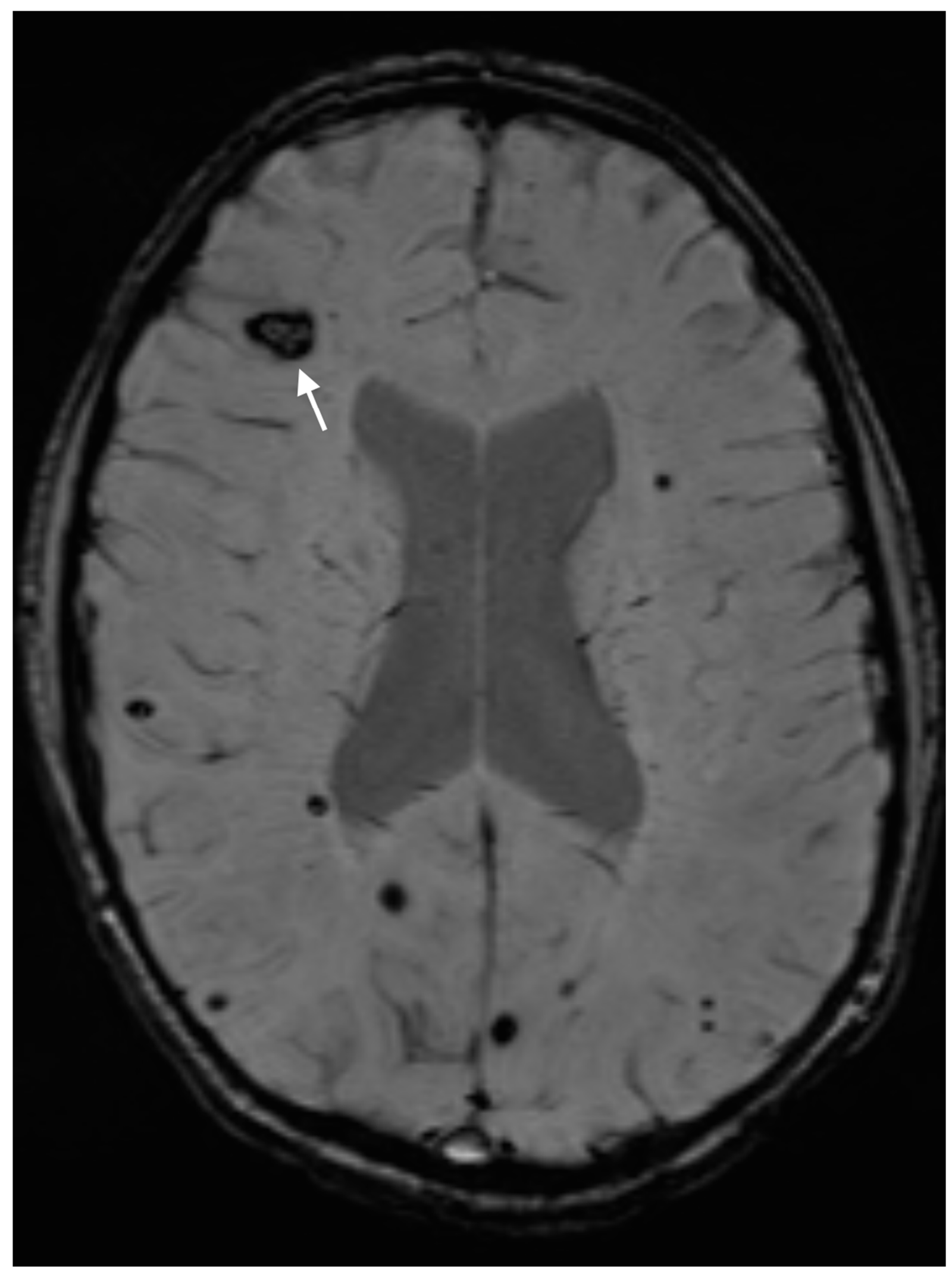




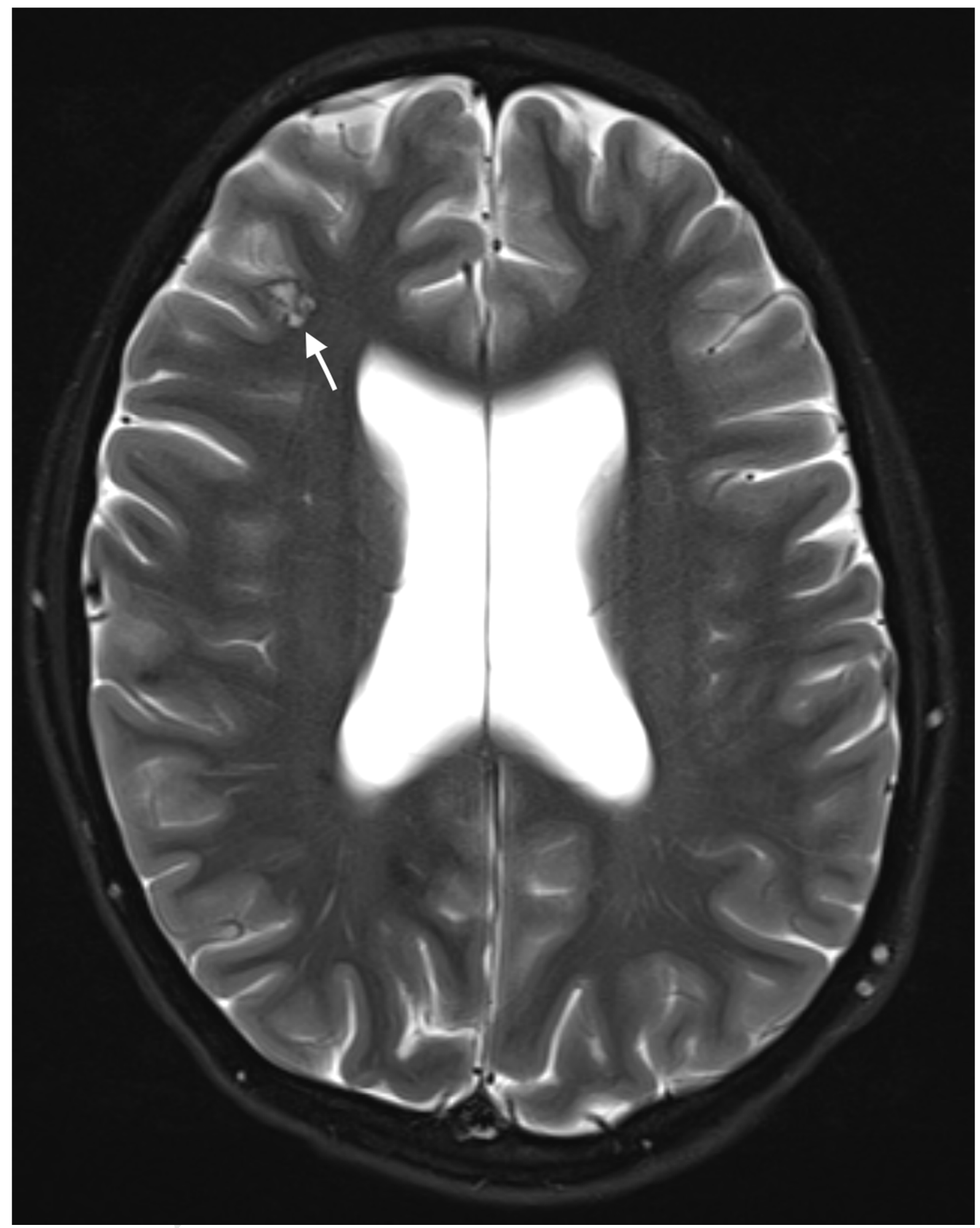


ACCEPTED MANUSCRIPT

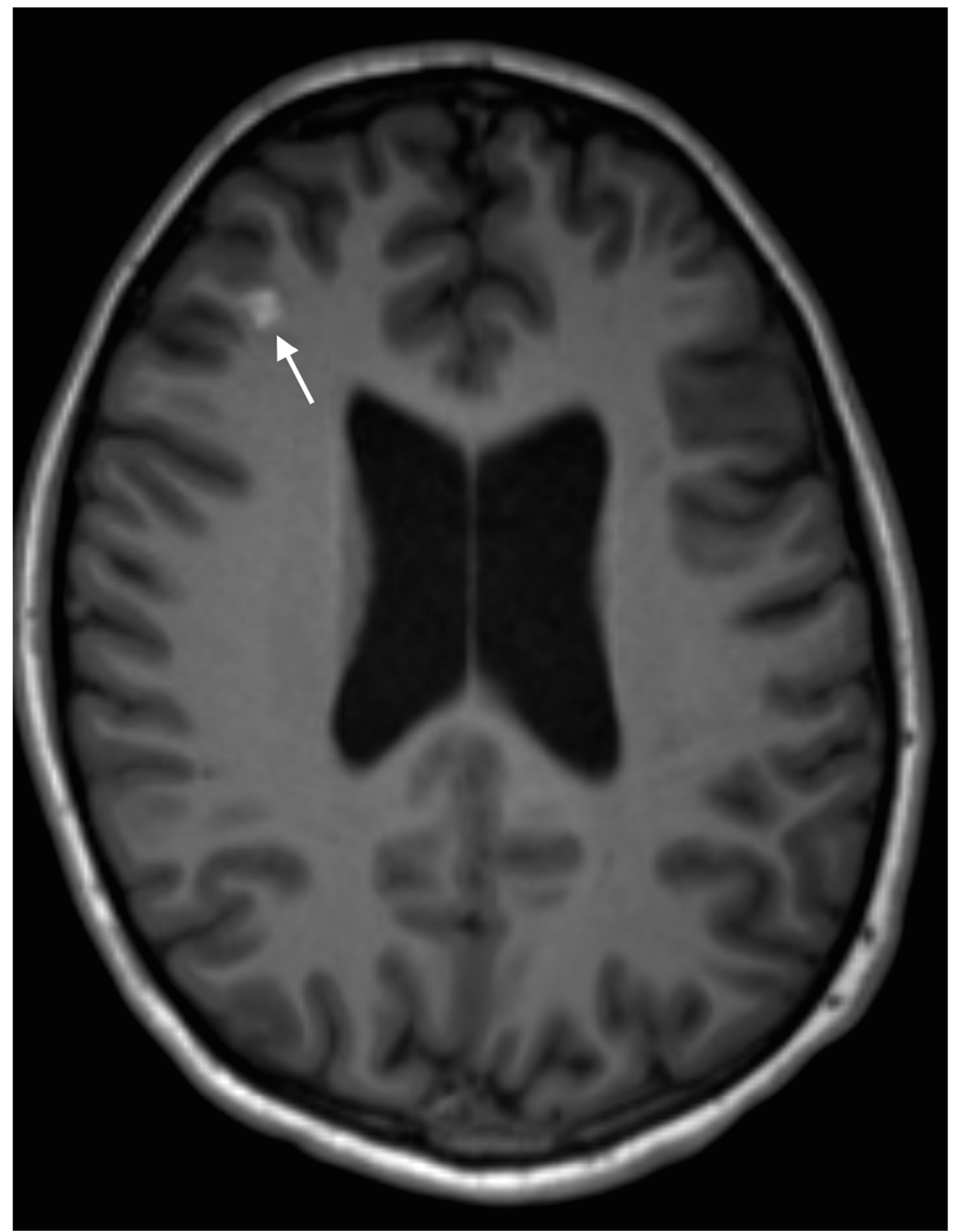




\section{ACCEPTED MANUSCRIPT}

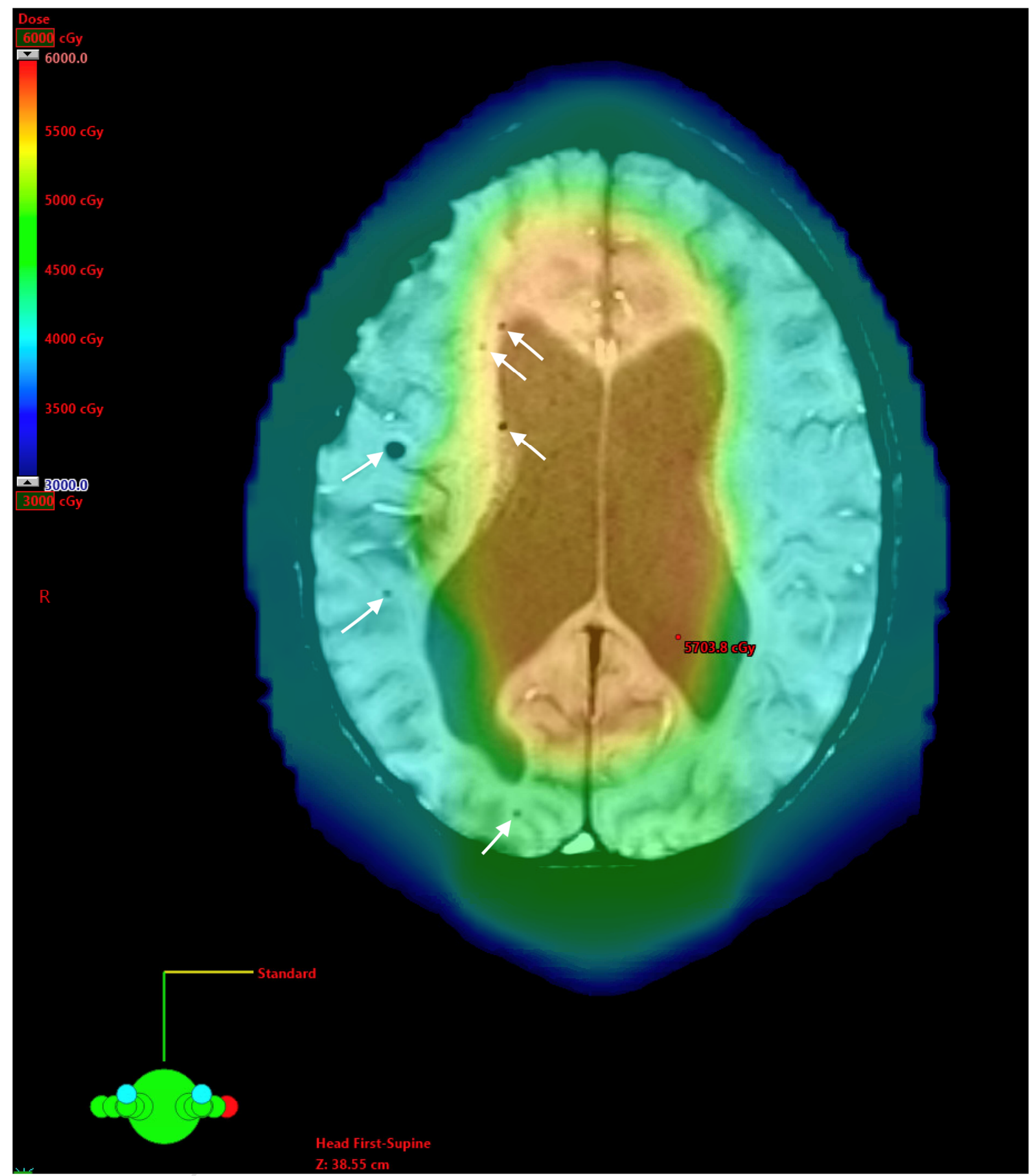

Z: $38.55 \mathrm{~cm}$ 


\section{SUMMARY:}

This study investigates radiation-induced cerebral microbleeds (CMBs) in pediatric patients with primary brain tumors treated with proton radiotherapy. This complication of radiotherapy has not been significantly studied following proton radiotherapy in the pediatric patient population, particularly with regards to timing, incidence and associated risk factors. CMBs have been associated with decreased neurocognitive function indicating the potential clinical significance. This research establishes an estimate for the timing and incidence of CMBs, evaluates how CMBs accrue over time, investigates risk factors associated with development of CMBs, and allows for a comparison between photon and proton radiotherapy. 